\title{
Band Structure of Phononic Crystal Consist of Hollow Aluminum Cylinders in Different Media; Finite Element Analysis
}

\author{
Zafer Ozer', Mehtap Demir ${ }^{2}$, Amirullah M. Mamedov ${ }^{3,4}$, Ekmel Ozbay $^{4}$ \\ ${ }^{1}$ Mersin Vocational High School Electronic and Automation Department, Mersin \\ University (Turkey) \\ ${ }^{2}$ Faculty of Engineering, Department of Metallurgy and Material Engineering, \\ Adiyaman University (Turkey) \\ ${ }^{3}$ Nanotechnology Research Center (NANOTAM), Bilkent University (Turkey) \\ ${ }^{4}$ International Scientific Center, Baku State University, Baku (Azerbaijan) \\ E-mail: zaferozer@mersin.edu.tr
}

\begin{abstract}
In this study, we investigated band structure of a 2D Phononic Crystal (PnC) which was consist of hollow aluminum cylinders in different media. To validate the finite element predictions, we made a square lattice PnC which has lattice constant $a=14 \mathrm{~mm}$, outer radius $r_{l}=5 \mathrm{~mm}$ and inner radius $r_{2}=4 \mathrm{~mm}$ of cylinders. In our experimental measurement, by using a signal generator software, we sent $100 \mathrm{~Hz}-20 \mathrm{kHz}$ sine signal for 5 seconds duration from speaker to the PnC where placed $1 \mathrm{~m}$ away from. With the help of a microphone, we recorded the sound in front of the PnC $\left(P_{i n}\right)$ and backside the PnC $\left(P_{\text {out }}\right)$. Then we were converted our recording into frequency domain by standard FFT algorithm, we calculated the Transmission Loss value according to the formula $T L=20 * \log _{10}\left(P_{\text {out }} / P_{\text {in }}\right)$ and obtained the experimental Transmission Loss. After experimental validation, we conducted band structure analysis of different combination of the $2 \mathrm{D} \mathrm{PnC}$ by finite element method.
\end{abstract}

\section{Introduction}

Phononic Crystals are periodic structures made of two elastic materials with different mechanical properties. The basic property of $\mathrm{PnC}$ is that mechanical (either elastic or acoustic) waves, having frequencies within a specific range, are not able to propagate within the periodic structure. This range of forbidden frequencies is called a phononic band gap. This is analogous to electrons in a crystal, where classical waves propagate in a structure with periodically modulated material parameters [1]. In recent years, many researchers have studied PnC. This is important for applications, such as acoustic filter, noise control, and subwavelength imaging $[2,3]$. To acquire wide acoustic band gaps at some desired frequency ranges, several factors are mainly considered, including scatter shapes, filing fraction, shear modulus ratio, mass density ratio, Poisson's ratio, and lattice structures [4].

In this study, we experimentally demonstrate the existence of complete band gaps in 2D phononic crystal consisting of a square array parallel aluminum cylinders in different media. The Finite Element Method (FEM) is used to calculate the band structure of phononic crystal. Experimental measured data are obtained and compared with the range of frequencies of complete band gaps.

\section{Materials and experiments}

In the PnC, the propagation of acoustic Bloch waves can be expressed by the acoustic wave equation as in Eq. (1). 
IOP Conf. Series: Materials Science and Engineering 613 (2019) 012018 doi:10.1088/1757-899X/613/1/012018

$$
\nabla \cdot\left(\frac{\mathbf{1}}{\boldsymbol{\rho}} \nabla p\right)=-\frac{1}{\rho c^{2}}[\omega(\tilde{k})]^{2} \cdot p
$$

where, $c=c(\tilde{r})$ is longitudinal velocity of acoustic waves inside the PnC, $\rho=\rho(\tilde{r})$ is density of the material and $p=p_{k}(\tilde{r})$ is the spatial part of $p_{k}(\tilde{r}, t)$ an acoustic Bloch wave within the PnC with wavevector $\tilde{k}$.

In figure 1a the basic vectors of the square lattice are $a_{1}=a \vec{x}, a_{2}=a \vec{y}$ and reciprocal lattice vectors in figure $1(\mathrm{~b})$ are $b_{1}=\frac{2 \pi}{a} \vec{x}, b_{2}=\frac{2 \pi}{a} \vec{y}$. In reciprocal space high symmetry points are; $\Gamma=(0,0)$, $X=\left(\frac{\pi}{a}, 0\right), M=\left(\frac{\pi}{a}, \frac{\pi}{a}\right)$.

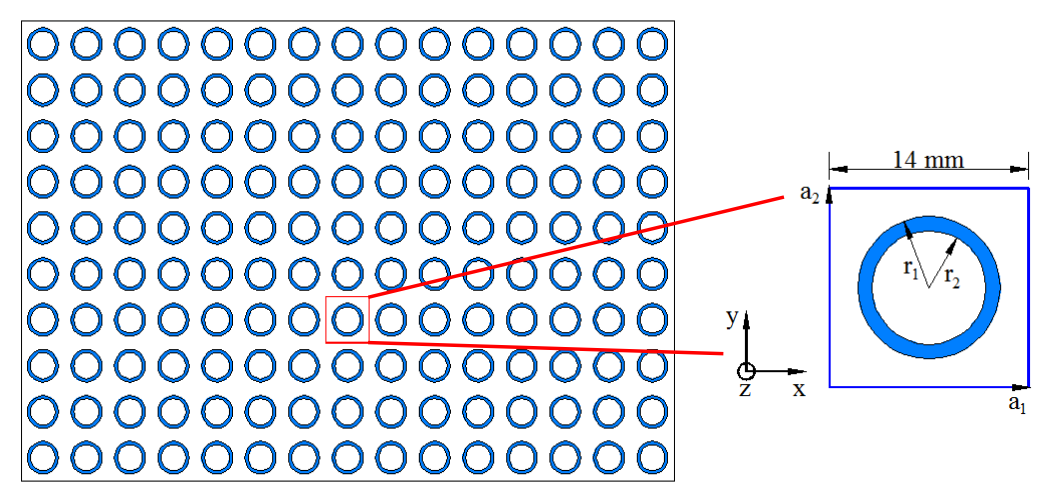

a)

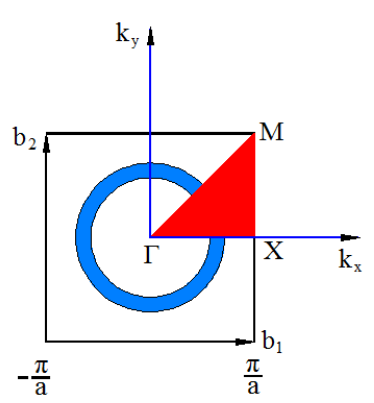

b)

Figure 1. a) Phononic crystal in rectangular lattice in the direct space and unit cell b) high symmetry points $(\Gamma-X-M)$ irreducible Brillouin Zone in reciprocal space

To obtain the band structure of PnC we applied Floquet periodic conditions edges of the unit cell in figure 1(a). The obtained acoustic band structure was plotted along the $M-\Gamma-X-M$ path for the two dimensional (2D) PnC as seen in fig. 2.

For validating the attenuation of acoustic waves as seen in band structure of unit cell, we conclude a measurement setup by finite array of $\mathrm{PnC}$ as seen in fig 1(a) and we designed the finite array as seen in fig. 2. In finite array FEM analysis, we applied Symmetry Boundary Conditions upper and lower edges, we assumed Perfectly Matched Layer left and right ends, and we applied 1 Pa pressure to the back pressure field in $x$ direction as seen in fig. 2. In fig.2 the points $A$ and $B$ are $P_{\text {in }}$ and $P_{\text {out }}$ observation points in following $T L$ formula.

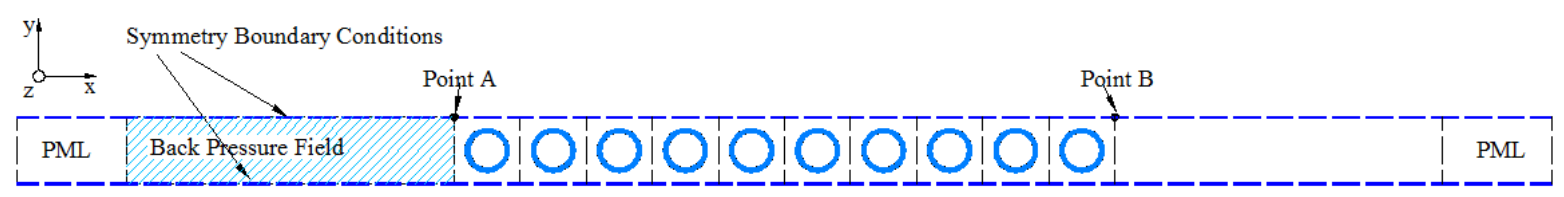

Figure 2. Boundary conditions of finite array FEM model

The experimental setup consists of 150 aluminum cylinders with an internal diameter of $8 \mathrm{~mm}$ an external diameter of $10 \mathrm{~mm}$ and length of $29.5 \mathrm{~mm}$ in the form of square lattice with a lattice constant $a=14 \mathrm{~mm}$ were placed in the holes on the fiber substrate in a 10x15 array.

In our experimental study, we placed a speaker $1 \mathrm{~m}$ away from the $\mathrm{PnC}$ and we sent a sound signal from $100 \mathrm{~Hz}$ to $20000 \mathrm{~Hz}$ on to the PnC for 5 seconds. With the microphone we placed behind the $\mathrm{PnC}$, we recorded the sound signal to the computer via an external sound card and converted it to the frequency domain with the standard FFT algorithm. Then we obtained the experimental transmission loss according to the formula $T L=20 * \log _{10}\left(P_{\text {out }} / P_{\text {in }}\right)$ where $P_{\text {in }}$ the data obtained from the microphone that we placed on the front side of the PnC and $P_{\text {out }}$ the data we obtained from the microphone which we placed behind the PnC. 
IOP Conf. Series: Materials Science and Engineering 613 (2019) 012018 doi:10.1088/1757-899X/613/1/012018

Furthermore, we performed parametric scanning to examine the effect of the thickness of the aluminum cylinder on the band structure by FEM. The band structure of PnC is calculated, with the geometrical parameters $r_{1}=5 \mathrm{~mm}, r_{2}=4,3.5,3,2.5 \mathrm{~mm}$ where $r_{1}$ and $r_{2}$ are the outer and inner radius of the hollow cylinder of large size, respectively.

\section{Results and discussions}

Obtained acoustic band structure in Brillouin zone of the square lattice was plotted along the $\mathrm{M}-\Gamma-\mathrm{X}-$ $\mathrm{M}$ path for the two dimensional (2D) $\mathrm{PnC}$ as seen in fig. 3, the narrow full band observed in the dispersion curves between 13.2-13.8 kHz and a partial band between $8-14 \mathrm{kHz}$ in $\Gamma$-X direction.

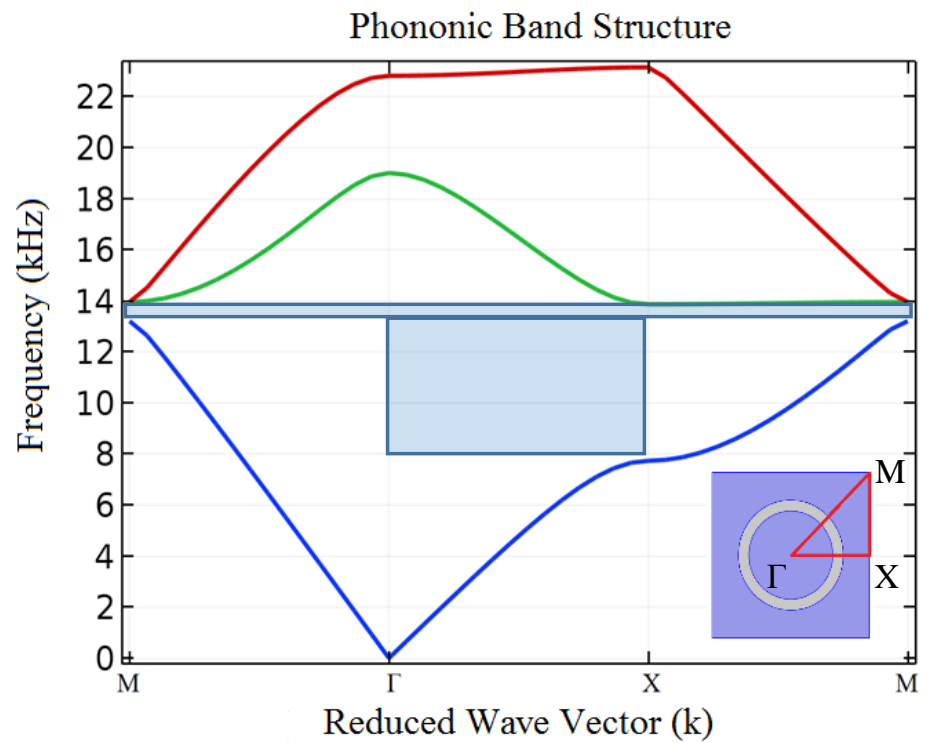

Figure 3. Acoustic band structure of $\mathrm{PnC}$

In order to compare the band structure obtained by FEM and the sound attenuation obtained by $\mathrm{PnC}$, transmission loss was obtained numerically with finite structure seen in Fig. 2 by FEM and experimental measurement. Fig.3 shows the numerical and experimental measurement results.

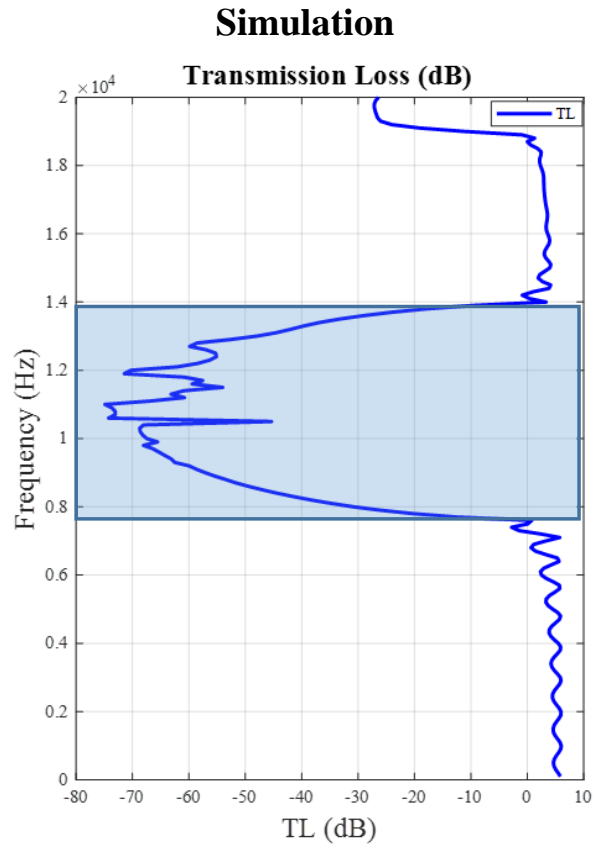

a)

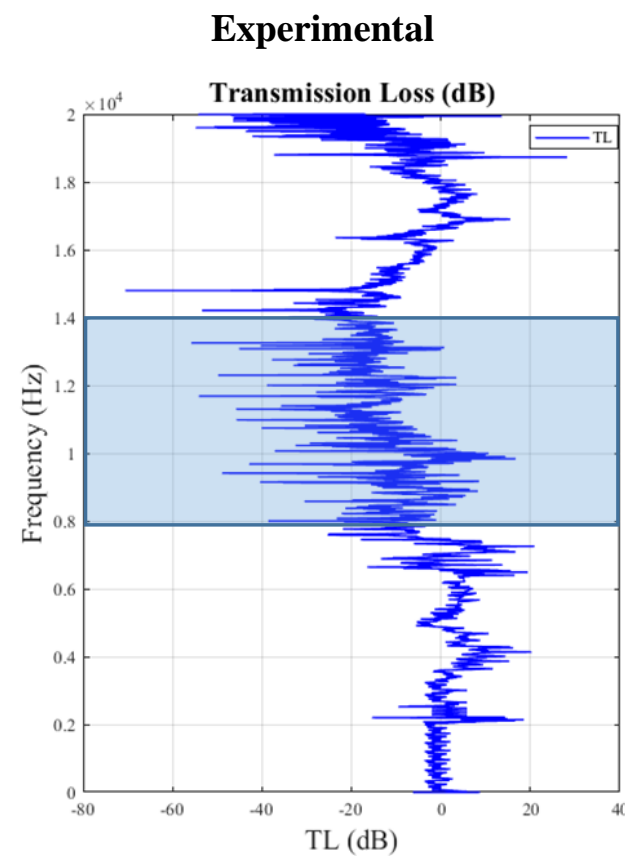

b)

Figure 4. Transmission Loss of finite array a) FEM analysis b) measurement 
According to the results of the transmission loss we obtained by FEM and experimental measurement, the sound level decreased between $8-14 \mathrm{kHz}$ as in the band structure and the results are compatible.

We investigated the effect of wall thickness (r1-r2) on the band gap using FEM. as shown in table 1, the center frequency and bandwidth are reduced when the wall thickness is increased.

Table 1. Band relation with wall thickness

\begin{tabular}{|c|c|c|}
\hline $\boldsymbol{r}_{\boldsymbol{I}} \boldsymbol{r}_{2}[\mathbf{m m}]$ & Central frequency $[\mathrm{Hz}]$ & Band Gap [Hz] \\
\hline 1 & 13543.5 & 661 \\
\hline 1.5 & 13540.5 & 659 \\
\hline 2 & 13538.5 & 657 \\
\hline 2.5 & 13537.5 & 657 \\
\hline 3 & 13537 & 656 \\
\hline
\end{tabular}

\section{Conclusions}

In the present paper, a PnC with hollow cylinder columns was presented and investigated. The band structure was calculated using the finite element method. The band gap and frequency can be tuned by changing the inner radius of the hollow cylinders. It could be based on the design of the elastic acoustic wave guides that transmit at tunable frequencies using proposed structures. We have also shown the band structure of the $2 \mathrm{D}$ PnC that is compatible with the transmission losses obtained by numerical and experimental measurement.

\section{References}

[1] P Deymier 2013 Acoustic Metamaterials and Phononic Crystals Springer-Verlag Berlin Heidelberg

[2] M S Kushwaha, P Halevi 1996 Applied Physics Letters 69 (1) 31 https://aip.scitation.org/doi/10.1063/1.118108

[3] A Sukhovich, B Merheb, K Muralidharan, J O Vasseur, Y Pennec, P A Deymier, J H Page 2009 Physical Review Letters 102 (15) 154301 https://doi.org/10.1103/PhysRevLett.102.154301

[4] C He, H Zhao, R Wei; B Wu 2010 Frontiers of Mechanical Engineering in China 5 (4) 450 https://doi.org/10.1007/s11465-010-0105-y 